ERRATUM

\title{
A brain drain
}

\section{Yvonne Bordon}

Nature Reviews Immunology 15, 404 (2015)

The original version of this article contained a typo in the final paragraph and incorrectly stated that interstitial fluid is originally drained from the CSF via the lymphatic system - the article has been amended to indicate that interstitial fluid from the brain parenchyma is initially drained into the CSF via the glymphatic system. 